TRANSACTIONS OF THE

AMERICAN MATHEMATICAL SOCIETY

Volume 351, Number 7, Pages 2623-2641

S 0002-9947(99)02141-8

Article electronically published on March 10, 1999

\title{
VAUGHT'S CONJECTURE AND THE GLIMM-EFFROS PROPERTY FOR POLISH TRANSFORMATION GROUPS
}

\author{
GREG HJORTH AND SLAWOMIR SOLECKI
}

\begin{abstract}
We extend the original Glimm-Effros theorem for locally compact groups to a class of Polish groups including the nilpotent ones and those with an invariant metric. For this class we thereby obtain the topological Vaught conjecture.
\end{abstract}

\section{Preface}

In this paper we consider equivalence relations induced by Polish groups acting continuously on Polish spaces. We improve on

0.1 Theorem (Sami). The topological Vaught conjecture holds for abelian Polish groups.

and extend to a class of groups much wider than abelian the dichotomy theorem established by Glimm and Effros in the locally compact case. In approximate order of presentation, the main results are:

0.2 Theorem. Let $G$ be a nilpotent Polish group acting continuously on a Polish space. Then there are either only countably many orbits or $2^{\aleph_{0}}$ many (in fact, perfectly many).

This strengthens 0.1 .

Let $E_{0}$ be the Vitali-like equivalence relation on $2^{\mathbb{N}}$ given by $x E_{0} y \Leftrightarrow \exists N \in$ $\mathbb{N} \forall n>N(x(n)=y(n))$.

0.3 Theorem. Let $G$ be a Polish group admitting an invariant metric and acting continuously on a Polish space $X$. Then either the orbit equivalence relation is $G_{\delta}$, or there is a continuous one to one function $f: 2^{\mathbb{N}} \rightarrow X$ such that

$$
\forall x, y \in 2^{\mathbb{N}} x E_{0} y \Leftrightarrow \exists g \in G(g \cdot f(x)=f(y)) .
$$

The second alternative here is actually equivalent to the existence of a $G$-ergodic Borel probability measure that gives every orbit measure zero. This second alternative also implies that the orbit equivalence relation Borel reduces the Vitali equivalence relation, in the sense that there is a Borel function $f: \mathbb{R} \rightarrow X$ such that

$$
\forall x, y \in \mathbb{R}((x-y) \in \mathbb{Q} \Leftrightarrow \exists g \in G(g \cdot f(x)=f(y))) .
$$

Received by the editors August 18, 1995 and, in revised form, June 16, 1997.

1991 Mathematics Subject Classification. Primary 04A15.

Key words and phrases. Polish group, orbit equivalence relation.

(C)1999 American Mathematical Society 
Indeed, the reader may choose to view this as being a dichotomy result regarding the Vitali equivalence relation - either the orbits are all $G_{\delta}$, or the action is as complicated as the Vitali equivalence relation given by $x E y$ if and only if $x-y \in \mathbb{Q}$. That formulation essentially captures the content of 0.3 .

The class of Polish groups admitting an invariant metric properly includes those that are abelian; therefore we obtain 0.3 for abelian Polish groups. However, in many ways this is already a surprising result, since it is known that for $G$ abelian Polish there are continuous actions on Polish spaces where the induced equivalence relation is not even Borel.

0.3 extends the above mentioned result by Glimm and Effros. As a corollary one obtains the topological Vaught conjecture for $G$ with an invariant metric, thereby answering a question raised in [Ke2], and again improving on 0.1 .

0.4 Theorem. Let $G$ be a nilpotent Polish group acting continuously on a Polish space $X$. Then either the orbit equivalence relation is $G_{\delta}$, or there is a continuous one to one function $f: 2^{\mathbb{N}} \rightarrow X$ such that

$$
\forall x, y \in 2^{\mathbb{N}} x E_{0} y \Leftrightarrow \exists g \in G(g \cdot f(x)=f(y)) .
$$

Again, this extends the work from [Gl], and again it can be used to prove the topological Vaught conjecture for nilpotent groups. However, 0.2 is given an independent proof in $\S 2$, since the proof there works for $\Sigma_{1}^{1}$ sets, as did Sami's theorem

for abelian groups [Sa], and in fact gives a kind of transfer result: for $Z(G)$ the center of $G$, if $G / Z(G)$ satisfies the topological Vaught conjecture on $\sum_{1}^{1}$ sets, then so too does $G$.

0.5 Corollary. Let $G$ and $X$ be as in 0.3 or 0.4 . Then for no $x \in X$ and infinite

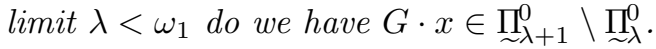

So in particular, for Polish groups in this class, we obtain that no orbit can be $\prod_{\lambda+1}^{0}$ complete. It is known in the case of more complicated Polish group actions that $\prod_{\lambda+1}^{0}$ complete orbits are possible.

A completely different type of corollary follows for minimal actions - those in which every orbit is dense.

0.6 Corollary. Let $G$ and $X$ be as in 0.3 or 0.4 , and suppose $x, y \in X$ have distinct orbits with the same closure. Then there are $2^{\aleph_{0}}$ many orbits. In particular, if the action is minimal and there is more than one orbit, then there are $2^{\aleph_{0}}$ many.

This corollary is analogous to a theorem by Vaught from model theory that states that no complete first order theory can have exactly two non-isomorphic models. As discussed in [Be] or 4.3 below, we can view the process of passing from a countable model to its theory as being one of assigning to a corresponding orbit its closure with respect to a suitable topology. Thus, Vaught's theorem states that a certain type of action cannot have exactly two orbits with a given closure, while 0.6 implies much stronger results for $G$ as above. Again it is known that 0.6 fails for general Polish groups; see for instance 4.1, or compare 4.4 for an example that makes no appeal to model theory.

We in fact prove 0.3 and 0.4 for an entirely general class of Polish groups that includes nilpotent, locally compact, and invariantly metrizable. In $\S 4$ we show that 
these Glimm-Effros dichotomies cannot be extended to solvable Polish groups or Polish groups with a complete left invariant metric.

0.7 Theorem. There is a rank two solvable Polish group $G$, acting continuously on a Polish space $X$, such that:

(i) $G$ has a complete left invariant metric;

(ii) the action is minimal;

(iii) there are 2 exactly orbits;

(iv) the conclusion of 0.3 and 0.4 fails.

Introductory remarks can be found in $\S 1$, where we recall some definitions and basic facts. We will assume at least a passing familiarity with descriptive set theory, along the lines of [Ke1]. In particular, we will make use of the Borel and projective hierarchies; the definitions of these hierarchies, as with other notions not defined in the course of the paper, can be found in [Ke1] and [Mo].

We thank Alexander Kechris - not only for writing [Ke1], but also for a number of helpful and thought provoking conversations on the subject matter of this paper, at all stages of its development. We also owe gratitude to the referee for a penetrating and thorough reading of this paper.

\section{BACKGROUND AND NOTATION}

Recall that a topological space is said to be Polish if it is separable and it admits a complete metric. It is a well known fact that any $G_{\delta}$ subset of a Polish space is again Polish; see for instance 3.11 of [Ke1]. $(X, S)$ is a standard Borel space if there is a topology $\tau$ on $X$ such that $X$ becomes a Polish space with respect to $\tau$ and $S$ is the $\sigma$-algebra of Borel sets for $(X, \tau)$.

1.1 Definition. Let $X$ be a Polish space and $E \subseteq X \times X$ be an equivalence relation. $E$ has perfectly many classes if there is a perfect set $P \subseteq X$ such that $\forall x, y \in P(x E y \Rightarrow x=y)$. We let $[x]_{E}=_{\text {df }}\{z \in X: z E x\}$ denote the equivalence class of $x \in X . E_{0} \subseteq 2^{\mathbb{N}} \times 2^{\mathbb{N}}$ is given by the specification that $x E_{0} y$ if and only if $\exists M \in \mathbb{N} \forall n>M(x(n)=y(n))$. For $E$ and $F$ both equivalence relations, on $X$ and $Y$ respectively, we write $E \sqsubseteq_{c} F$ if there is a continuous injective function $f: X \hookrightarrow Y$ such that

$$
\forall x, y \in X(x E y \Leftrightarrow f(x) F f(y)) .
$$

It turns out that for Borel, or even $\prod_{1}^{1}$, equivalence relations one can prove an analog of the perfect set theorem for Borel sets.

1.2 Theorem (Silver). Let $X$ be Polish and $E \subseteq X \times X a \prod_{1}^{1}$ equivalence relation.

Then $E$ has either perfectly many classes or at most countably many. ( $\square$ See [Si])

It is shown in [HaKeLo] that a very different kind of dichotomy theorem can be proved for Borel equivalence relations, having no parallel with any of the classical perfect set theorems: Let $E$ be a Borel equivalence relation (on a Polish space $X$ ); then either $E_{0} \sqsubseteq_{c} E$ or there is a Borel function $\theta: X \rightarrow 2^{\omega}$ such that

$$
\forall x, y \in X(x E y \Leftrightarrow \theta(x)=\theta(y)) .
$$

This result built on earlier work of [Gl] and [Ef] that showed a stronger result under the additional assumption that $E$ is induced by the continuous action of a locally compact group; see 1.6 below. 
1.3 Definition. $G$ is a Polish group if it is a topological group that is Polish as a topological space. A metric $d$ is said to be left invariant if $\forall g_{0}, g_{1} \in G d\left(g_{0}, g_{1}\right)=$ $d\left(g_{1}^{-1} g_{0}, 1\right)$, and right invariant if $\forall g_{0}, g_{1} \in G d\left(g_{0}, g_{1}\right)=d\left(g_{0} g_{1}^{-1}, 1\right)$; it is simply said to be invariant if it is both left and right invariant.

It is well known, and can be found in [BeKe2], that any invariant metric on a Polish group $G$, compatible with the topology on $G$, is necessarily complete. It also follows by the same types of arguments that any abelian Polish group has a compatible invariant metric; indeed, it is natural to view the property of having an invariant metric as a topological approximation to the algebraic property of being abelian. A weaker notion is that of having a compatible complete left invariant metric. Here one can show that all locally compact Polish groups have a left invariant complete metric, but not in general an invariant metric. To put the later results in context, the reader should probably also be aware that not all nilpotent Polish groups admit an invariant metric. An all-purpose counterexample was pointed out by Alexander Kechris: Let $G=\left\{\left(a_{i j}\right)_{i, j \leq 3} \in S L(3, \mathbb{R}): a_{i i}=1, i \leq\right.$ $\left.3 ; a_{i j}=0, j>i\right\}$; this is locally compact and nilpotent, but there is no invariant metric.

If $G$ is Polish and $H \triangleleft G$ is closed, then it follows that $G / H$ is again a Polish group in the quotient topology; again, the reader can find a proof in [BeKe2]. If $G$ is a Polish group and $X$ is a Polish space equipped with some continuous action by $G$, then we will say that $X$ is a Polish $G$-space. If $X$ is a standard Borel space equipped with a Borel action by $G$, then we will say it is a Borel $G$-space. Here we mean that the action is Borel with respect to some Polish topology $\tau$, compatible with $X$, in the sense that it is a Borel subset of $G \times X \times X$ in the product topology one obtains from $\tau$. We shall normally avoid all explicit mention of the action itself and draw no distinction between the Polish $G$-space and the underlying Polish space on which the action takes place.

Given $G$ acting on $X$, in either of the two contexts above, we will let $\cdot$ denote the action, so that for $g \in G, x \in X, g \cdot x$ denotes the result of applying $g$ to $x$. If $A \subseteq G$ and $B \subseteq X$, then we set $g \cdot B={ }_{\mathrm{df}}\{g \cdot y: y \in B\}, A \cdot x={ }_{\mathrm{df}}\{h \cdot x: h \in A\}$, and $A \cdot B=\{h \cdot y: h \in A, y \in B\}$. We will say that a set $B \subseteq X$ is $G$-invariant, or, when the context makes clear, invariant, if $G \cdot B=B$. For $x \in X, G_{x}={ }_{\mathrm{df}}\{g \in$ $G: g \cdot x=x\}$ is the stabilizer of $x ; G_{x}$ is always closed (clearly trivial in the case of continuous actions, this last fact was proved for Borel actions in [MiD]). $G \cdot x$ is called the orbit of $x$. We let $E_{G}^{X} \subseteq X \times X$ be the orbit equivalence relation, given by $x E_{G}^{X} y \Leftrightarrow \exists g \in G(g \cdot x=y)$.

For $G$ an arbitrary group, $Z(G)$ denotes the center of $G$, which is the set $\{g \in$ $G: \forall h \in G(h \cdot g=g \cdot h)\}$ of elements that commute with all of $G$. $G$ is said to be nilpotent if there is a sequence $Z_{k}=G \triangleright Z_{k-1} \triangleright \cdots Z_{0}=\{1\}$, for some $k \in \mathbb{N}$, such that $Z_{n+1} / Z_{n}=Z\left(G / Z_{n}\right)$ for each $n<k$.

The topological Vaught conjecture states that if $G$ is a Polish group and $X$ is a Polish $G$-space, then either $X$ has at most $\aleph_{0}$ many orbits or $E_{G}^{X}$ has a perfect set of equivalence classes. The topological Vaught conjecture for $G$ is the statement that this dichotomy holds for any Polish $G$-space. Although this paper contains some partial results for particular choices of $G$, the general conjecture remains totally open. Note that by 1.2 , the conjecture is trivially satisfied when $E_{G}^{X}$ is Borel, as is a weak version of the Glimm-Effros property. However, in general it can be $\sum_{1}^{1}$ and non-Borel, and it is known that there can be examples of $\sum_{1}^{1}$ equivalence 
relations with exactly $\aleph_{1}$ many classes; therefore, any general proof of Vaught's conjecture must, like the arguments in this paper, do more than appeal to the logical complexity of $E_{G}^{X}$. We should also note that since [So] shows that even for abelian Polish $G$ we can have $E_{G}^{X}$ non-Borel, the special cases of Vaught's conjecture proved in $\S 2$ and $\S 3$ cannot be reduced to 1.2 .

1.5 Definition. Let $G$ be a Polish group. We will say that $G$ has the Glimm-Effros property if whenever $X$ is a Polish $G$-space, either $E_{0} \sqsubseteq_{c} E_{G}^{X}$ or $E_{G}^{X} \in G_{\delta}$.

1.6 Theorem (Effros). If $G$ is Polish locally compact, then $G$ has the GlimmEffros property.

$(\square$ See $[$ Ef].)

We should point out that definition 1.5 is due to us, and neither Glimm nor Effros. It is well known and easly checked that the Glimm-Effros property for $G$ implies the topological Vaught's conjecture for $G$. Not all Polish groups have the Glimm-Effros property even in the weaker forms suggested by [HaKeLo]; the reader can find a counterexample in $[\mathrm{HjKe}]$, as well as a proof of a distantly related dichotomy theorem that holds for arbitrary Polish $G$. What one can prove is the following, which in turn becomes the basis for $\S 3$.

1.7 Theorem (Becker-Kechris). Let $G$ be a Polish group and $X$ a Polish $G$-space. Then either $E_{0} \sqsubseteq_{c} E_{G}^{X}$ or there is a $G_{\delta}$ orbit.

$(\square$ See $[\mathrm{BeKe} 2]$.)

1.7 should be compared with the following theorem. We will in fact use both results.

1.8 Theorem (Effros). Let $G$ be a Polish group and let $X$ be a Polish $G$-space. Then for any $x \in X$, the following three things are equivalent:

(i) $G \cdot x \in G_{\delta}$;

(ii) $G \cdot x$ is a Baire space - that is, non-meager in its own topology;

(iii) the natural map $G / G_{x} \rightarrow G \cdot x$, given by $h G_{x} \mapsto h \cdot x$, is a homeomorphism. ( $\square$ See $[\mathrm{Ef}]$.)

The other theorem by Becker and Kechris that we will need states that there is little difference between Polish $G$-spaces and Borel $G$-spaces when $G$ is a Polish group. This will be crucial for us in $\S 2$ when we complete the inductive step at 2.5 .

1.9 Theorem (Becker-Kechris). Let $G$ be a Polish group and $X$ a Borel $G$-space. Then there is a Polish topology for $X$, compatible with the Borel structure on $X$, such that $(X, \tau)$ is a Polish $G$-space.

$(\square$ See $[\mathrm{BeKe} 2]$.

The proof of 1.9 gives the following important corollary, also explicitly noted by Becker and Kechris.

1.10 Theorem (Becker-Kechris). Let $G$ be a Polish group and $X$ a Polish $G$-space. Let $\tau_{0}$ be the Polish topology on $X$. Let $A \subseteq X$ be invariant and ${\underset{\sim}{0}}_{\alpha}^{0}$, some $\alpha<\omega_{1}$.

Then there is a Polish topology $\tau_{1}$ on $X$, refining $\tau_{0}$, such that

(i) $A$ is clopen in $\tau_{1}$;

(ii) there is a countable basis $\mathcal{B}$ for $\tau_{1}$ such that every $U \in \mathcal{B}$ is $\sum_{\sim \alpha+n}^{0}$, with respect to $\tau_{0}$, for some $n \in \mathbb{N}$;

(iii) the action of $G$ on $X$ remains continuous with respect to the new topology. $(\square$ See $[\mathrm{BeKe} 2]$. 
It is a classical fact, recalled in [Sa], that a countable increasing sequence of Polish topologies again generates a Polish topology. In this respect one could envisage a situation where we apply 1.10 to a sequence $\left(A_{i}\right)_{i \in \mathbb{N}}$ of Borel sets, so that $A_{i}$ is closed in $\left(X, \tau_{i}\right), \tau_{0} \subseteq \tau_{1} \subseteq \cdots \tau_{i} \subseteq \tau_{i+1} \cdots$. Then in the topology $\tau_{\omega}$ generated by $\bigcup_{i \in \mathbb{N}} \tau_{i}$ each $A_{i}$ is clopen.

We will indeed need to perform this kind of construction in $\S 3$ when we obtain as a corollary of the main result that there are no properly $\prod_{\lambda+1}^{0}$ orbits in actions by nilpotent or invariantly metrizable groups. In order to lay the groundwork for this application, we recall the notion of Vaught transforms.

1.11 Notation. Let $G$ be a Polish group and $X$ a Borel $G$-space. Let $A \subseteq X$ be Borel and let $U \subseteq G$ be open. $A^{\Delta U}$ refers to the set of $x \in X$ such that for a non-meager collection of $g \in U$ we have $g \cdot x \in A$. $A^{* U}$ refers to the set of $x \in X$ such that for a co-meager in $U$ collection $g \in U$ we have $g \cdot \in A$. The sets $A^{* U}$ and $A^{\Delta U}$ are called Vaught transforms of $A$

It is shown in [Va] that for $X$ a Polish $G$-space, if $A$ is $\sum_{\alpha}^{0}$, then $A^{\Delta U}$ is $\sum_{\alpha}^{0}$, and if $A$ is $\prod_{\alpha}^{0}$ then $A^{* U}$ is $\prod_{\alpha}^{0}$. It is easily seen that $A^{\Delta G}$ and $A^{* G}$ are always invariant.

\section{VAught's COnjecture for nilpotent Polish groups}

In this section we show that the topological Vaught's conjecture holds for nilpotent Polish groups acting in a Borel fashion on a Polish space. Actually, what we really show is somewhat more general - both in covering a class of groups somewhat more general than nilpotent and in covering a class of spaces somewhat more general than Polish. It will be convenient to first give a definition and a lemma.

2.1 Definition. Let $\Gamma$ be some point class and let $G$ be some Polish group. Then $\operatorname{TVC}(G, \Gamma)$ indicates that whenever $X$ is a Borel $G$-space and $A \subseteq X$ is invariant and in $\Gamma$, then $A$ has either countably many or perfectly many orbits. We write $\operatorname{TVC}(G)$ for $\operatorname{TVC}(G$, Borel).

2.2 Lemma. Let $G$ be a Polish group and $X$ a Polish $G$-space. Suppose that $x, y \in X$ with $G \cdot x \in \prod_{\alpha}^{0}$. Suppose $x$ is in the closure of $Z(G) \cdot y$ and $y$ is in the closure of $Z(G) \cdot x$. Then $G \cdot y \in \underset{\sim \alpha+5}{\prod_{\alpha}^{0}}$.

Proof. First note the following general fact:

Claim: Suppose $\left(g_{i}\right)_{i \in \mathbb{N}},\left(h_{i}\right)_{i \in \mathbb{N}}$ are two sequences from $G$ and $A \subseteq X$ is $\underset{\sim}{\prod_{\beta}^{0}}$.

Then the set $\left\{a \in X: \exists z\left(g_{i} \cdot a \rightarrow z \wedge z \in A \wedge h_{i} \cdot z \rightarrow a\right)\right\}$ is $\prod_{\beta+5}^{0}$.

Proof of claim. Fix a complete metric $d$ on $X$. To say that $g_{i} \cdot a$ is Cauchy is $\prod_{\sim}^{0}$, and so the existence of a limit point is $\underset{\sim}{\prod_{3}^{0}}$. For $z$ the limit, we have that $a$ is the limit of $h_{i} \cdot z$ if and only if

$$
\forall k>0 \exists N \forall n \geq N \exists M \forall m \geq M\left(d\left(a, h_{m} \cdot g_{n} \cdot a\right) \leq 1 / k\right) .
$$

This is $\underset{\sim}{0}$. It is easily checked that for $C$ closed and $z=\lim g_{i} \cdot a$

$$
z \in C \Leftrightarrow \forall k>0 \exists n>k\left(d\left(C, g_{n} \cdot a\right)<1 / k\right) .
$$

This completes the proof of the claim in the case that $\beta=0$. Now the claim for arbitrary $C \in \prod_{\beta+5}^{0}\left(\beta<\omega_{1}\right)$ follows by induction on $\beta$.

$(\square$ claim) 
To finish the proof of the lemma, let us next suppose that $\left(g_{i}\right)_{i \in \mathbb{N}},\left(h_{i}\right)_{i \in \mathbb{N}}$ are included in the center of $G$, with $g_{i} \cdot y \underset{i}{\rightarrow} x$ and $h_{i} \cdot x \underset{i}{\rightarrow} y$. Then for any $g \in G$ we have

$$
g_{i} \cdot g \cdot y=g \cdot g_{i} \cdot y \underset{i}{\rightarrow} g \cdot x
$$

and

$$
h_{i} \cdot g \cdot x=g \cdot h_{i} \cdot x \underset{i}{\rightarrow} g \cdot y
$$

by the definition of the center and the continuity of the action. If we take $A=G \cdot x$, then the lemma follows from the claim.

We will also need an observation due to Ramez Sami.

2.3 Lemma (Sami). Let $G$ be a Polish group and $X$ a Borel $G$-space. Let $\alpha$ be a countable ordinal. Define $F$ to be an equivalence relation on $X$ given by

$$
x F y \Leftrightarrow \forall A \in \prod_{\alpha}^{0}(A=G \cdot A \Rightarrow(x \in A \Leftrightarrow y \in A)) .
$$

Then $F$ is $\prod_{1}^{1}$

We refer the reader to [Sa] for the proof. It might be worth remarking, however, that 2.3 is proved by the use of Vaught transforms and the fact that it is uniformly Borel in the code for $B$, a $\prod_{\alpha}^{0}$ set, to say that $x \in B^{* G}$

2.4 Corollary (Sami). Let $G$ be a Polish group and $X$ a Borel $G$-space. Let $\alpha$ be a countable ordinal and $A \subseteq X$ a $\Sigma_{1}^{1}$ set. Suppose that $A$ does not have a perfect set of $E_{G}^{X}$-inequivalent reals, but that for all $x \in A, G \cdot x \in \prod_{\alpha}^{0}$. Then $E_{G}^{X}$ has at most $\aleph_{0}$ many equivalence classes in $A$.

Proof. Since any $\sum_{1}^{1}$ set is the Borel image of a Borel set, let $A=f[B]$, where $B$ is a Borel set and $f$ is a Borel function. Define $E$ on $B$ by $x E y$ if and only if $f(x) E_{G}^{X} f(y)$. $E$ is $\prod_{\sim}^{1}$, by 2.3 , so if $E$ has more than $\aleph_{0}$ many equivalence classes then it follows by Silver's theorem for $\prod_{1}^{1}$ equivalence relations that there will be some perfect set of $E$-inequivalent reals, $B_{0} \subset B$. Then its image under $f$ must again contain a perfect set by the perfect set theorem for $\sum_{1}^{1}$ (see [Ke, p. 226]).

Keep in mind that $Z(G)$, the center of $G$, is closed in any topological Hausdorff group. Therefore, if $G$ is Polish, so is $G / Z(G)$.

2.5 Theorem. If $G$ is a Polish group, then $\operatorname{TVC}\left(G / Z(G), \Sigma_{\sim 1}^{1}\right)$ implies $\operatorname{TV} C\left(G, \Sigma_{\sim}^{1}\right)$.

Proof. Suppose $\operatorname{TVC}\left(G / Z(G), \Sigma_{1}^{1}\right)$, and let $X$ be a Borel $G$-space. Let $A \subseteq X$ be $\sum_{1}^{1}$ with less than perfectly many orbits. We assume $A$ has exactly $\aleph_{1}$ many orbits and try to find a contradiction. Following [BeKe1] or [BeKe2], we can equip $X$ with a compatible topology $\tau$ so that $\tau$ generates the original Borel structure on $X$ and $(X, \tau)$ is a Polish $G$-space. For $x \in X$, let $F(x)=\overline{Z(G) \cdot x}$, with the topological closure taken in $(X, \tau)$. Let $F(X, \tau)$ be the Borel space of closed sets in $(X, \tau)$ - the fact that this is a standard Borel space is discussed in [Ke, p. 75]. $F: X \rightarrow F(X, \tau)$, $x \mapsto F(x)$ is Borel, since if we fix a countable dense subgroup $G_{0} \subset G$, then for 
all open $U \subset X$ we have $U \cap F(x) \neq \emptyset \Leftrightarrow \exists g \in G_{0}(g \cdot x \in U)$ by continuity of the action. For $F \in F(X, \tau)$ and $h \in G$, let $h \cdot F=\{h \cdot z: z \in F\}$ be the translation of $F$ by $h$. It is easily seen that $F(X, \tau)$ becomes a Borel $G$-space under this action.

Claim (i): For $x \in X$, and $h \in G, h \cdot F(x)=F(h \cdot x)$.

Proof of claim. Note that $h \cdot Z(G) \cdot x=Z(G) \cdot h \cdot x$ by the definition of $Z(G)$ as the center. Then $h \cdot \overline{Z(G) \cdot x}=\overline{Z(G) \cdot h \cdot x}$ by continuity of the action. ( $\square$ claim)

Claim (ii): $\exists D \subseteq F(X, \tau), D \supseteq\{F(x): x \in X\}, D \in \Delta_{\sim}^{1}, D G$-invariant, such that

$$
\forall g \in Z(G) \forall F \in D(g \cdot F=F) .
$$

Proof of claim. Let $A_{0}=\{F(x): x \in X\}$ and $B=\{F \in F(X, \tau): \exists g \in$ $Z(G)(g \cdot F \neq F)\}$. Note that $A_{0}$ and $B$ are disjoint $\Sigma_{\sim 1}^{1}$ sets by the definition of the function $x \mapsto F(x)$. So we can find $D_{0} \in \Delta_{\sim}^{1}, D_{0} \supseteq A_{0}, D_{0} \cap B=\emptyset$. Let $A_{1}=\left\{h \cdot F: h \in G, F \in D_{0}\right\}$ be the saturation of $D_{0}$ under the $G$ action. Since $B$ is $G$-invariant, $A_{1} \cap B=\emptyset$. Repeating the argument, we find that $D_{0} \subseteq A_{1} \subseteq D_{1} \subseteq A_{2} \subseteq \cdots \subseteq A_{i} \subseteq D_{i} \subseteq \cdots$ with each $D_{i} \in \Delta_{1}^{1}$, each $A_{i} \in \Sigma_{1}^{1}$ and $G$-invariant, with $A_{i} \cap B=\emptyset$. Then $D=\bigcup_{i} D_{i}$ is as required.

$(\square$ claim)

Thus $D$ is a Borel $G$-space. In some natural sense it is also a Borel $G / Z(G)$ space, since if $h_{1} \cdot Z(G)=h_{2} \cdot Z(G)$, say $h_{1} \cdot g_{0}=h_{2}$ for $g_{0} \in Z(G)$, then for any $F \in D$

$$
h_{2} \cdot F=\left(h_{1} \cdot g_{0}\right) \cdot F=h_{1} \cdot\left(g_{0} \cdot F\right)=h_{1} \cdot F .
$$

Let $A^{*}=\{F(x) \in F(X, \tau): x \in A\}$. Note that $A^{*}$ is $\sum_{\sim 1}^{1}$ and $G / Z(G)$-invariant by claim (i). Again by claim (i) and the assumption that $A$ has less than perfectly many orbits, it must be the case that $A^{*}$ has at most $\aleph_{0}$ many orbits, in light of $\operatorname{TVC}\left(G / Z(G), \Sigma_{1}^{1}\right)$ applied to $D$ viewed as a Borel $G / Z(G)$-space.

So we can assume without loss of generality that $A^{*}$ consists of just one orbit, so that for all $x, y \in A$,

$$
F(x) E_{G / Z(G)}^{D} F(y)
$$

in other words,

$$
\exists g \in G \overline{Z(G) \cdot(g \cdot x)}=\overline{Z(G) \cdot y} .
$$

But now if we fix a single $y \in A$ and suppose that $G \cdot y \in \prod_{\sim \alpha}^{0}$, then it follows from this and 2.2 that for all $x \in A$ we have $G \cdot x \in \prod_{\sim \alpha+5}^{0}$. Hence, by $2.4, E_{G}^{X}$ has at most $\aleph_{0}$ many equivalence classes in $A$ after all.

2.6 Corollary. If $G$ is nilpotent Polish, then $\operatorname{TVC}\left(G, \Sigma_{\sim 1}^{1}\right)$.

Proof. By induction on the length of the central series. In the trivial case of $G=\{1\}$ the result follows from the perfect set theorem for $\sum_{1}^{1}$ (see [Ke]). The inductive step follows by 2.5 .

It is worth mentioning in passing that the proof of 2.5 will work for pointclasses more general than $\sum_{1}^{1}$ in the presence of determinacy assumptions. For instance, if PD (the axiom of Projective Determinacy) holds, then for any nilpotent Polish $G$ 
and $n \in \mathbb{N}$ we obtain $\operatorname{TVC}\left(G, \sum_{n}^{1}\right)$. The point is that if $X$ is a Borel $G$-space and $A \subseteq X$ is $\sum_{n}^{1}$, with fewer than perfectly many orbits, then by the argument in 2.5 and our inductive assumption of $\operatorname{TVC}\left(G / Z(G), \sum_{\sim}^{1}\right)$, we obtain that there is some $\gamma<\omega_{1}$ such that $\forall x \in A\left(G \cdot x \in \underset{\sim}{\prod_{\gamma}^{0}}\right)$. Since $A$ has fewer than perfectly many orbits, we can well-order the equivalence classes by Theorem 5 of [HaSa, p. 258]; but by the PD version of Theorem 4.5 of [Ha, p. 690] there is no projective $\aleph_{1}$-sequence of $\prod_{\sim}^{0}$ sets, so $A$ can have at most $\aleph_{0}$ many orbits.

As another application, 2.5 gives $\operatorname{TVC}\left(G \times H, \underset{\sim 1}{\Sigma_{1}^{1}}\right)$ whenever $G$ is locally compact and $H$ is nilpotent. It follows from 2.4 and Silver's theorem that we must have $\operatorname{TVC}\left(G, \Sigma_{1}^{1}\right)$, where $G$ is locally compact.

\section{The Glimm-Effros property}

In this section we prove that if $G$ is nilpotent Polish or Polish with an invariant metric, $G$ satisfies the Glimm-Effros property: If $X$ is a Polish $G$-space, and $E_{G}^{X}$ is the induced orbit equivalence relation, then either

I) $E_{G}^{X} \in G_{\delta}$; or

II) $E_{0} \sqsubseteq c E_{G}^{X}$.

Glimm originally proved this for $G$ and $X$ locally compact, and this was later extended by Effros to the case of $G$ Polish and $E_{G}^{X} \in F_{\sigma}$. However, there is one important difference between that case and this one. Here our assumptions on the action are not sufficient to guarantee that $E_{G}^{X}$ should be Borel, and for this reason we found the discovery of the Glimm-Effros property, even for $G$ abelian Polish, to be unexpected. So while it follows from the results of [So] that $E_{G}^{X}$ may be non-Borel in the case of $G$ abelian, it follows by the results here that this only happens when the orbit structure is extremely complicated - for instance, there is an $E_{G}^{X}$-ergodic Borel probability measure that takes the value zero on each orbit. (See $[\mathrm{HjKe}]$ for discussion of when such measures exist.)

Another consequence of the results in this section is that no minimal action of a Polish abelian group can have exactly two orbits or even exactly $\alpha$ many for $2 \leq \alpha \leq \aleph_{0}$; recall that an action is minimal if every orbit is dense. This is reminiscient of Vaught's theorem that no countable complete first order theory can have precisely two models up to isomorphism. The analogy is suggested by the observation, from say [Be], that the set of countable models with a given first order theory corresponds exactly to the set of orbits in a Polish $S_{\infty}$-space that have a corresponding closure in the topology generated by first order logic.

A further consequence of the results below that has no meaningful parallel in the locally compact case is in the direction of calculating possible Borel complexities of a single orbit. While in the locally compact case we must have every orbit $F_{\sigma}$, in the abelian case there is no such bound on the possible complexity. For this reason it is perhaps somewhat surprising to discover that if $G$ is abelian, or nilpotent, or with an invariant metric, and $X$ is a Polish $G$-space, then for no $x \in X, \lambda$ a countable limit ordinal, can $G \cdot x$ be properly $\prod_{\lambda+1}^{0}$. It is known that in the case of more complicated Polish groups, such as $S_{\infty}$, one can have properly $\prod_{\lambda+1}^{0}$ orbits; see [Mi]. 
Finally, as the Glimm-Effros property implies Vaught's conjecture, the results below imply Vaught's conjecture for Polish groups with an invariant metric. This is easily checked, since if $E_{0} \sqsubseteq_{c} E_{G}^{X}$, then a routine argument provides a perfect set of $E_{G}^{X}$-inequivalent reals, while if $E_{G}^{X}$ is $G_{\delta}$, or even just Borel, then [Si] shows that there are either $\leq \aleph_{0}$ many orbits or a perfect set of inequivalent reals. In either case, Vaught's conjecture holds. In fact, one obtains Vaught's conjecture for the more general class of groups isolated in the statement of 3.1.

Before giving 3.1, it might be helpful to recall the definition of the central series for a group $G$. Let $Z_{0}=\{1\}$, and let $Z_{k+1}$ be the pre-image of the center of $G / Z_{k}$ by the natural homomorphism $G \rightarrow G / Z_{k}$. The sequence $\left(Z_{k}\right)_{k \in \mathbb{N}}$ is the central series of $G$. Each $Z_{k}$ is a normal subgroup of $G$, and when $G$ is a Hausdorff topological group we have that each $Z_{k}$ is closed.

3.1 Theorem. Let $G$ be a Polish group, and let $\left(Z_{k}\right)_{k \in \mathbb{N}}$ be its central series. If there is $k_{0} \in \mathbb{N}$ such that $G / Z_{k_{0}}$ admits an invariant metric or is locally compact, then $G$ has the Glimm-Effros property.

It is worth remarking that there are two important classes of groups covered by the theorem above: Polish groups admitting an invariant metric (take $k_{0}=0$ ), and nilpotent Polish groups (choose $k_{0}$ least so that $Z_{k_{0}}=G$ ). We will need to prove some lemmas before returning to the proof of 3.1.

3.2 Lemma. Let $X$ be a Polish $G$-space, $G$ a Polish group. Let $H \triangleleft G$ be closed. Let $x_{0}, y \in X$. Assume that

(i) $\overline{G \cdot x_{0}}=\overline{G \cdot y}$ and $G \cdot x_{0}$ is $G_{\delta}$;

(ii) $G / H$ admits a complete left invariant metric that is right invariant with respect to elements of $G_{x_{0}} / H$ or $G / H$ is locally compact.

Then there is $x_{1} \in G \cdot x_{0}$ such that $y \in \overline{H \cdot x_{1}}$.

Proof. First, we observe that it is enough to find $g_{i} \in G, i \in \mathbb{N}$, with $g_{i} x_{0} \underset{i}{\longrightarrow} y$ and $g_{i} / H \underset{i}{\longrightarrow} g / H$ for some $g \in G$. Indeed, if this holds, we can find $g_{i}^{H} \in H$ with $g_{i} g_{i}^{H} \underset{i}{\longrightarrow} g$. Let $\epsilon_{i} \underset{i}{\longrightarrow} 1$ be such that $\epsilon_{i} g_{i} g_{i}^{H}=g$ for each $i \in \mathbb{N}$. By the normality of $H, \epsilon_{i} g_{i} g_{i}^{H}=\bar{g}_{i}^{H} \epsilon_{i} g_{i}$ for some $\bar{g}_{i}^{H} \in H$. Since $g_{i} \cdot x_{0} \underset{i}{\longrightarrow} y$ and $\epsilon_{i} \underset{i}{\longrightarrow} 1$, we have $\epsilon_{i} g_{i} x_{0} \rightarrow y$. Thus $\left(\bar{g}_{i}^{H}\right)^{-1} \cdot\left(g \cdot x_{0}\right) \underset{i}{\longrightarrow} y$. Take $x_{1}=g \cdot x_{0}$, as required.

Now, assume that $G / H$ is locally compact. Let $U \subseteq G$ be open and such that $U / H$ has compact closure in $G / H$. By Effros' Theorem, $U \cdot x_{0}$ is an open subset of $G \cdot x_{0}$. Since $G \cdot x_{0}$ is a dense $G_{\delta}$ in $\overline{G \cdot y}, \overline{U \cdot x_{0}} \cap G \cdot y \neq \emptyset$. So, pick $g_{i} \in U, i \in \mathbb{N}$ and $g_{\infty} \in G$ with $g_{i} \cdot x_{0} \underset{i}{\longrightarrow} g_{\infty} \cdot y$. We can assume that $\left(g_{i} / H\right)_{i \in \mathbb{N}}$ converges, since $\overline{U / H}$ is compact. But then $\left(g_{\infty}^{-1} g_{i} / H\right)_{i \in \mathbb{N}}$ converges as well, and $g_{\infty}^{-1} g_{i} \cdot x_{0} \underset{i}{\longrightarrow} y$.

Now, let $G / H$ admit a left invariant complete metric which is right invariant with respect to elements of $G_{x_{0}} / H$. Let $g_{i}, h_{i} \in G$, for $i \in \mathbb{N}$, be such that $g_{i} \cdot x_{0} \underset{i}{\longrightarrow} y$ and $h_{i} \cdot y \underset{i}{\longrightarrow} x_{0}$. Let $\rho$ be a metric on $X$. By taking subsequences we can assume that $\rho\left(h_{i} \cdot y, x_{0}\right)<\frac{1}{2(i+1)}$. Given $i \in \mathbb{N}$, for sufficiently large $k$ we have $\rho\left(h_{i} g_{k} \cdot x_{0}, h_{i} \cdot y\right)<\frac{1}{2(i+1)}$. So again by passing to a subsequence we may assume that $\rho\left(h_{i} g_{j} \cdot x_{0}, h_{i} \cdot y\right)<\frac{1}{2(i+1)}$ for $j \geq i$. Thus $\rho\left(h_{i} g_{j} \cdot x_{0}, x_{0}\right)<\frac{1}{i+1}$ for all $j \geq i$.

Let $d_{H}$ be a metric on $G / H$ as in the hypothesis. Let $\left\{V_{i}: i \in \mathbb{N}\right\}$ be a neighborhood basis at 1 for the topology on $G$, with $d_{H}$-diam $\left(V_{i} / H\right)<1 / 2^{i+1}-$ 
i.e., for all $g, h \in V_{i}, d_{H}(g / H, h / H)<\frac{1}{2^{i+1}}-\epsilon$, for some fixed $\epsilon>0$. By Effros' theorem, each $V_{i} \cdot x_{0}$ is open in $G \cdot x_{0}$, so, by taking subsequences as necessary, we can assume that $h_{i} g_{j} \cdot x_{0} \in V_{i} \cdot x_{0}$ for $j \geq i$. Thus there are sequences $c_{i}^{0}, c_{i}^{1} \in G_{x_{0}}, i \in \mathbb{N}$, such that $h_{i} g_{i} c_{i}^{0}, h_{i} g_{i+1} c_{i}^{1} \in V_{i}$. Therefore

$$
d_{H}\left(h_{i} g_{i} c_{i}^{0} / H, h_{i} g_{i+1} c_{i}^{1} / H\right)<\frac{1}{2^{i}} .
$$

So by the left invariance of $d_{H}$,

$$
d_{H}\left(g_{i} c_{i}^{0} / H, g_{i+1} c_{i}^{1} / H\right)<1 / 2^{i} .
$$

But now by the right invariance of $d_{H}$ with respect to elements in $G_{x_{0}} / H$ we get

$$
d_{H}\left(g_{i} / H, g_{i+1} c_{i}^{1}\left(c_{i}^{0}\right)^{-1} / H\right)<1 / 2^{i} .
$$

Put $c_{0}=1$ and $c_{i+1}=c_{i}^{1}\left(c_{i}^{0}\right)^{-1}$. It follows from the right invariance of $d_{H}$ with respect to $G_{x_{0}} / H$ that the sequence $\left(g_{i} c_{i} c_{i-1} \cdots c_{0} / H\right)_{i \in \mathbb{N}}$ is $d_{H}$-Cauchy. Note that $g_{i} c_{i} c_{i-1} \cdots c_{0} \cdot x_{0} \underset{i}{\longrightarrow} y$. So, without loss of generality, after replacing $g_{i}$ by $g_{i} c_{i} c_{i-1} \cdots c_{0}$, we may assume that $\left(g_{i} / H\right)_{i \in \mathbb{N}}$ is $d_{H}$-Cauchy. Thus there exists $g \in G$ with $g_{i} / H \underset{i}{\longrightarrow} g / H$, since $d_{H}$ is a complete metric.

3.3 Lemma. Let $G$ be a Polish group and let $X$ be a Polish $G$-space. Let $H \triangleleft G$. Assume $G \cdot x \in G_{\delta}, x \in \overline{G \cdot y}$, and $y \in \overline{H \cdot x}, x, y \in X$. Then $x \in \overline{\left\langle H, G_{x}\right\rangle \cdot y}$.

Proof. Let $h_{i} \in H, g_{i} \in G, i \in \mathbb{N}$, be such that $h_{i} \cdot x \underset{i}{\longrightarrow} y$ and $g_{i} \cdot y \underset{i}{\longrightarrow} x$. As in the proof of Lemma 3.2, by taking subsequences we can assume that $g_{i} h_{i} \cdot x \underset{i}{\longrightarrow} x$. By Effros' theorem, there are $\epsilon_{i} \underset{i}{\longrightarrow} 1$ and $c_{i} \in G_{x}$ such that $\epsilon_{i} g_{i} h_{i}=c_{i}$. By the normality of $H$, we have $c_{i}=\epsilon_{i} g_{i} h_{i}=\bar{h}_{i} \epsilon_{i} g_{i}$ for some $\bar{h}_{i} \in H$. Thus, $\bar{h}_{i}^{-1} c_{i} \cdot y=$ $\epsilon_{i} g_{i} \cdot y \underset{i}{\longrightarrow} x$ as $\epsilon_{i} \underset{i}{\longrightarrow} 1$ and $g_{i} \cdot y \underset{i}{\longrightarrow} x$. Since $\bar{h}_{i}^{-1} c_{i} \in\left\langle H, G_{x}\right\rangle$, we are done.

3.4 Lemma. Let $G$ be a Polish group with the central series $\left(Z_{k}\right)_{k \in \mathbb{N}}$. Let $H<G$ be closed. Then $\overline{\left\langle Z_{k-1}, H\right\rangle} \triangleleft \overline{\left\langle Z_{k}, H\right\rangle}$, and $\overline{\left\langle Z_{k}, H\right\rangle} / \overline{\left\langle Z_{k-1}, H\right\rangle}$ is abelian.

Proof. By continuity, it is enough to check that $\left\langle Z_{k-1}, H\right\rangle \triangleleft\left\langle Z_{k}, H\right\rangle$ and that $\left\langle Z_{k}, H\right\rangle /\left\langle Z_{k-1}, H\right\rangle$ is abelian. Assume $g \in\left\langle Z_{k-1}, H\right\rangle$ and $h \in\left\langle Z_{k}, H\right\rangle$ and $g=$ $z_{0} c_{0}, h=z_{1} c_{1}$ with $z_{0} \in Z_{k-1}, z_{1} \in Z_{k}, c_{0}, c_{1} \in H$. Then for some $\bar{z}_{0} \in Z_{k-1}$ we have

$$
h g h^{-1}=z_{1} c_{1} z_{0} c_{0} c_{1}^{-1} z_{1}^{-1}=z_{1} z_{1}^{-1} \bar{z}_{0} c_{1} c_{0} c_{1}^{-1}=\bar{z}_{0} c_{1} c_{0} c_{1}^{-1},
$$

since $Z_{k} / Z_{k-1}$ is the center of $G / Z_{k-1}$. Thus, $h g h^{-1} \in\left\langle Z_{k-1}, H\right\rangle$, whence

$$
\left\langle Z_{k-1}, H\right\rangle \triangleleft\left\langle Z_{k}, H\right\rangle \text {. }
$$

Now, let $\bar{g}_{1}, \bar{g}_{2} \in\left\langle Z_{k}, H\right\rangle /\left\langle Z_{k-1}, H\right\rangle$, and let $g_{1}, g_{2} \in Z_{k}, c_{1}, c_{2} \in H$ be such that $\bar{g}_{1}=\pi\left(g_{1} c_{1}\right)$ and $\bar{g}_{2}=\pi\left(g_{2} c_{2}\right)$, where $\pi:\left\langle Z_{k}, H\right\rangle \rightarrow\left\langle Z_{k}, H\right\rangle /\left\langle Z_{k-1}, H\right\rangle$ is the projection. Then, for some $c \in H, z, z^{\prime} \in Z_{k-1}$,

$$
\bar{g}_{2} \bar{g}_{1}=\pi\left(g_{2} c_{2} g_{1} c_{1}\right)=\pi\left(g_{1} g_{2} c_{1} c_{2} c z\right)=\pi\left(g_{1} c_{1} g_{2} c_{2} c z^{\prime}\right)=\bar{g}_{1} \bar{g}_{2} .
$$

Thus, $\left\langle Z_{k}, H\right\rangle /\left\langle Z_{k-1}, H\right\rangle$ is abelian.

3.5 Lemma. Let $G$ and $k_{0}$ be as in 3.1. Let $X$ be a Polish $G$-space. Assume $x, y \in X$ with $\overline{G \cdot x}=\overline{G \cdot y}$ and $G \cdot x \in G_{\delta}$. Then $G \cdot x=G \cdot y$.

Proof. We will recursively construct $x_{k} \in X$, for $k=k_{0}, k_{0}-1, \cdots, 0$, so that

(i) $x_{k} \in G \cdot x$; 
(ii) $y \in \overline{Z_{k} \cdot x_{k}}$.

This will prove the lemma, since for $k=0$, (ii) gives $y=x_{0}$ and (i) gives $x_{0} \in G \cdot x$, whence $G \cdot x=G \cdot y$.

By Lemma 3.2 , there is $x_{k_{0}} \in G \cdot x$ with $y \in \overline{Z_{k_{0}} \cdot x_{k_{0}}}$. Therefore, we can produce $x_{k}$ for $k=k_{0}$, as required to begin the construction.

Now assume $x_{k}$ as required has been produced. Put $Z^{*}=\overline{\left\langle Z_{k}, G_{x_{k}}\right\rangle}$. We want to apply Lemma 3.2 to get $x_{k-1} \in Z^{*} \cdot x_{k}$ with $y \in \overline{\left\langle Z_{k-1}, G_{x_{k}}\right\rangle \cdot x_{k-1}}$. Since $Z_{k} \triangleleft G$, by Lemma 3.3 and the inductive assumption (ii), we obtain $\overline{Z^{*} \cdot x_{k}}=\overline{Z^{*} \cdot y}$. Note that $Z^{*} / G_{x_{k}}$ is closed in $G / G_{x_{k}}$ as $Z^{*}=\pi^{-1}\left[Z^{*} / G_{x_{k}}\right]$, where $\pi: G \rightarrow G / G_{x_{k}}$ is the projection, and $Z^{*}$ is closed. Therefore, by Effros' theorem, we see that $Z^{*} \cdot x_{k}$ is relatively closed in $G \cdot x_{k}$, whence $Z^{*} \cdot x_{k} \in G_{\delta}$. By Lemma $3.4, \overline{\left\langle Z_{k-1}, G_{x_{k}}\right\rangle} \triangleleft Z^{*}$ and the Polish group $Z^{*} / \overline{\left\langle Z_{k-1}, G_{x_{k}}\right\rangle}$ is abelian, and so certainly admits an invariant metric. Hence, by Lemma 3.2, there is $x_{k-1} \in Z^{*} \cdot x_{k}$ with $y \in \overline{\left\langle Z_{k-1}, G_{x_{k}}\right\rangle \cdot x_{k-1}}$.

Then clearly (i) holds for $x_{k-1}$. To check (ii), it suffices to prove that $G_{x_{k}} \subseteq$ $\overline{\left\langle Z_{k-1}, G_{x_{k-1}}\right\rangle}$, since then

$$
y \in \overline{\left\langle Z_{k-1}, G_{x_{k}}\right\rangle \cdot x_{k-1}} \subseteq \overline{\overline{\left\langle Z_{k-1}, G_{x_{k-1}}\right\rangle} \cdot x_{k-1}}=\overline{Z_{k-1} \cdot x_{k-1}} .
$$

Now $x_{k-1} \in Z^{*} \cdot x_{k}$ implies that there are $z_{i} \in Z_{k}, c_{i} \in G_{x_{k}}$ and $z \in Z^{*}$ such that $x_{k-1}=z \cdot x_{k}$ and $c_{i} z_{i} \underset{i}{\longrightarrow} z$. Note that $z G_{x_{k}} z^{-1}=G_{x_{k-1}}$, and $z_{i}^{-1} z=\bar{z}_{i} z z_{i}^{-1}$ for some $\bar{z}_{i} \in Z_{k-1}$. Thus, since Lemma 3.4 gives $\overline{\left\langle Z_{k-1}, G_{x_{k-1}}\right\rangle} \triangleleft \overline{\left\langle Z_{k}, G_{x_{k-1}}\right\rangle}$, we obtain

$$
z_{i}^{-1} z G_{x_{k}} z^{-1} z_{i}^{-1}=z_{i}^{-1} G_{x_{k-1}} z_{i} \subseteq z_{i}^{-1} \overline{\left\langle Z_{k-1}, G_{x_{k-1}}\right\rangle} z_{i}=\overline{\left\langle Z_{k-1}, G_{x_{k-1}}\right\rangle}
$$

Also

$$
z_{i}^{-1} z G_{x_{k}} z^{-1} z_{i}=\bar{z}_{i} z\left(c_{i} z_{i}\right)^{-1} G_{x_{k}}\left(c_{i} z_{i}\right) z^{-1} \bar{z}_{i}^{-1}
$$

Hence

$$
z\left(c_{i} z_{i}\right)^{-1} G_{x_{k}}\left(c_{i} z_{i}\right) z^{-1} \subseteq \overline{\left\langle Z_{k-1}, G_{x_{k-1}}\right\rangle} \text { for all } i \in \mathbb{N}
$$

It then follows that $G_{x_{k}} \subseteq \overline{\left\langle Z_{k-1}, G_{x_{k-1}}\right\rangle}$ as $c_{i} z_{i} \underset{i}{\longrightarrow} z$.

Proof of Theorem 3.1. Define the equivalence relation $F$ on $X$ by $x F y$ iff $\overline{G \cdot x}=$ $\overline{G \cdot y}$. Then $F$ is $G_{\delta}$ since $x F y$ iff $\forall n\left(G \cdot x \cap V_{n} \neq \emptyset \Leftrightarrow G \cdot y \cap V_{n} \neq \emptyset\right)$ iff $\forall n\left(x \in G \cdot V_{n} \Leftrightarrow y \in G \cdot V_{n}\right)$ for an open basis $\left\{V_{n}: n \in \mathbb{N}\right\}$ for the topology on $X$. Clearly $E_{G}^{X} \subseteq F$. If $E_{G}^{X}=F, E_{G}^{X}$ is $G_{\delta}$. If $E_{G}^{X} \neq F$, then for some $x \in X$ the invariant set $[x]_{F}$ contains at least two orbits. Since $[x]_{F}$ is $G_{\delta}$ and each orbit contained in it is dense in it, it follows from Lemma 3.5 and the Becker-Kechris theorem that $E_{0} \sqsubseteq_{c} E_{G}^{X} \mid[x]_{F}$. Thus $E_{0} \sqsubseteq_{c} E_{G}^{X}$.

The next theorem shows that for a class of groups much wider than that considered in Theorem 3.1 (containing, in particular, all solvable Polish groups or all Polish groups admitting a complete left invariant metric) the Glimm-Effros dichotomy holds for continuous actions which are close to being free (all stabilizers are assumed to be compact). Important particular cases of this theorem were first noticed by Alexander Kechris after seeing the first draft of 3.1.

3.6 Theorem. Let $G$ be a Polish group with the property that there are closed subgroups $H_{i}<G, 0 \leq i \leq k$, such that $H_{0}=\{1\}, H_{k}=G, H_{i-1} \triangleleft H_{i}$, and 
$H_{i} / H_{i-1}$ has a complete left invariant metric. Let $X$ be a Polish $G$-space such that $G_{x}$ is compact for all $x \in X$. Then either $E_{G}^{X} \in G_{\delta}$ or $E_{0} \sqsubseteq_{c} E_{G}^{X}$.

Proof. If $E_{G}^{X}$ is not $G_{\delta}$, then, by an argument as in the proof of Theorem 3.1, we can assume that all orbits are dense and that there are at least two orbits. Now, if we show that no orbit is $G_{\delta}$, we will be done, since then by the Becker-Kechris theorem (Theorem 1.7) $E_{0} \sqsubseteq_{c} E_{G}^{X}$. Assume towards a contradiction that $G \cdot x \in G_{\delta}$ for some $x \in X$. The following claim will finish the proof.

Claim. Let $G$, which is as above, act continuously on a Polish space $X$ in such a way that each orbit is dense. Assume that, for some $x \in X, G \cdot x \in G_{\delta}$ and $G_{x}$ is compact. Then $G \cdot x=X$.

Proof of claim. Let $y \in X \backslash G \cdot x$. We will produce $x_{k}, x_{k-1}, \cdots, x_{0}$ such that

(i) $x_{k}=x, x_{i-1} \in H_{i} \cdot x_{i}$ for $1 \leq i \leq k$;

(ii) $\overline{H_{i} \cdot x_{i}}=\overline{H_{i} \cdot y}$ for $i \leq k$;

(iii) $H_{i} \cdot x_{i} \in G_{\delta}$ for $i \leq k$.

For $i=0$, (ii) gives $x_{0}=y$, which along with (i) contradict $y \notin G \cdot x$. Let $x_{k}=x$. Suppose $x_{i}$, with $1 \leq i \leq k$, have been constructed. Put $K=\left(G_{x_{i}} \cap H_{i}\right) / H_{i-1}$. Then $K$ is a compact subgroup of $H_{i} / H_{i-1}$, since $G_{x_{i}}$ is compact, being conjugate to $G_{x}$. Let $d$ be a complete left invariant metric on $H_{i} / H_{i-1}$. Define for $g_{1}, g_{2} \in$ $H_{i} / H_{i-1}$

$$
\tilde{d}\left(g_{1}, g_{2}\right)=\int_{K} d\left(g_{1} k, g_{2} k\right) d \mu(k)
$$

where $\mu$ is the normalized Haar measure on $K$. It is routine to check that $\tilde{d}$ is a complete left invariant metric on $H_{i} / H_{i-1}$ equivalent with $d$. Moreover, $\tilde{d}$ is right invariant with respect to elements of $K$. Note that $K=\left(H_{i}\right)_{x_{i}} / H_{i-1}$, as $G_{x_{i}} \cap H_{i}=\left(H_{i}\right)_{x_{i}}$. Thus, since from our inductive assumption we have $H_{i} \cdot x_{i} \in G_{\delta}$ and $\overline{H_{i} \cdot x_{i}}=\overline{H_{i} \cdot y}$, we can apply Lemma 3.2 and get $x_{i-1} \in H_{i} \cdot x_{i}$ with

$$
y \in \overline{H_{i-1} \cdot x_{i-1}} \text {. }
$$

Clearly (i) is fulfilled. By Lemma 3.3,

$$
x_{i-1} \in \overline{\left\langle H_{i-1}, G_{x_{i-1}} \cap H_{i}\right\rangle \cdot y} .
$$

Since $H_{i-1} \triangleleft H_{i}$

$$
\left\langle H_{i-1}, G_{x_{i-1}} \cap H_{i}\right\rangle=\left\{g h: h \in H_{i-1}, g \in G_{x_{i-1}} \cap H_{i}\right\} .
$$

So, there are $h_{n} \in H_{i-1}, g_{n} \in G_{x_{i-1}} \cap H_{i}$ with $g_{n} h_{n} \cdot y \underset{n}{\longrightarrow} x_{i-1}$. Since $G_{x_{i-1}} \cap H_{i}$ is compact, we can assume that $g_{n} \underset{n}{\longrightarrow} g \in G_{x_{i-1}}$. It follows that $g h_{n} \cdot y \underset{n}{\longrightarrow} x_{i-1}$, SO

$$
h_{n} \cdot y \underset{n}{\longrightarrow} g^{-1} \cdot x_{i-1}=x_{i-1} .
$$

Thus, we get $x_{i-1} \in \overline{H_{i-1} \cdot y}$, which combined with (*) gives (ii). To check (iii), note that since $H_{i} \cdot x_{i-1}=H_{i} \cdot x_{i} \in G_{\delta}$, the natural mapping $H_{i} /\left(G_{x_{i-1}} \cap H_{i}\right) \longrightarrow H_{i} \cdot x_{i-1}$ is a homeomorphism. Thus, since $\overline{\left\langle H_{i-1}, G_{x_{i-1}} \cap H_{i}\right\rangle} /\left(G_{x_{i-1}} \cap H_{i}\right)$ is closed in $H_{i} /\left(G_{x_{i-1}} \cap H_{i}\right)$, we have that $\overline{\left\langle H_{i-1}, G_{x_{i-1}} \cap H_{i}\right\rangle} \cdot x_{i-1} \in G_{\delta}$. But $H_{i-1} \triangleleft H_{i}$ and $G_{x_{i-1}} \cap H_{i}$ is compact, so

$$
\overline{\left\langle H_{i-1}, G_{x_{i-1}} \cap H_{i}\right\rangle}=\left\{h g: h \in H_{i-1}, g \in G_{x_{i-1}} \cap H_{i}\right\} .
$$


Thus,

$$
H_{i-1} \cdot x_{i-1}=\overline{\left\langle H_{i-1}, G_{x_{i-1}} \cap H_{i}\right\rangle} \cdot x_{i-1} \in G_{\delta} .
$$

Notice in particular that the hypothesis of 3.6 is satisfied when we know both that $G$ is solvable and that the action of $G$ on $X$ is free. We will see in $\S 4$ that neither of these assumptions by themselves is sufficient for the conclusion of 3.6.

Theorem 3.6, or rather its proof, implies that each solvable Polish group carries a left invariant complete metric. Recall that a group $G$ is solvable if there exist a finite sequence $H_{i}<G, i \leq k$, such that $H_{0}=\{1\}, H_{k}=G, H_{i-1} \triangleleft H_{i}$, and $H_{i} / H_{i-1}$ is abelian. Clearly if $G$ is a Polish solvable group, then we can assume that all the $H_{i}$ 's are closed. Note that then $H_{i} / H_{i-1}$ is a Polish abelian group, so it admits a left invariant (actually two-sided invariant) complete metric.

3.7 Corollary. Each Polish solvable group admits a left invariant complete metric compatible with the topology.

Proof. Let $d$ be a left invariant metric on $G$. Let $X$ be the completion of $G$ with respect to $d$. We will identify $G$ with a subset of $X$ in the natural fashion. We aim to show that $G=X$. Elements of $X$ are equivalence classes of $d$-Cauchy sequences of elements of $G$, where two $d$-Cauchy sequences $\left(g_{n}\right),\left(h_{n}\right)$ are identified if $\lim _{n} d\left(g_{n}, h_{n}\right)=0$. It is not difficult to check that if $\left(g_{n}\right)$ is $d$-Cauchy, then so is $\left(g g_{n}\right)$ for any $g \in G$ and, moreover, that if $\left(g_{n}\right)$ and $\left(h_{n}\right)$ represent the same point of $X$, then $\left(g g_{n}\right)$ and $\left(g h_{n}\right)$ represent the same point of $X$ as well. This allows us to define an action of $G$ on $X$ by letting $g \in G$ map the equivalence class of $\left(g_{n}\right)$ to the equivalence class of $\left(g g_{n}\right)$. The following claim holds for an arbitrary Polish group $G$. Checking it is quite straightforward, and we leave it to the reader.

Claim. (i) The action of $G$ on $X$ defined above is continuous.

(ii) The orbit of $1 \in G \subseteq X$ is homeomorphic to $G$, so it is a $G_{\delta}$ in $X$.

(iii) $G_{1}=\{1\}$, so the stabilizer of $1 \in G \subseteq X$ is compact.

(iv) Each orbit is dense.

Now if $G$ is Polish solvable, it fulfills the assumption of Theorem 3.6. Thus by the claim in the proof of Theorem 3.6 and the above claim, the orbit of 1 , which is $G \subseteq X$, is equal to $X$, that is, $G$ is equal to its $d$-completion, which can only mean that $d$ is complete.

The next proposition holds in the more general context of 3.1 , but we state it only for the special case of $G$ nilpotent or admitting an invariant metric.

3.8 Proposition. Let $G$ be a Polish group that is nilpotent or has an invariant metric. Let $X$ be a Polish $G$-space and let $x \in X$ be such that $G \cdot x \in \prod_{\lambda+1}^{0}$ for some countable infinite limit ordinal $\lambda$. Then there is a countable collection $\mathcal{B}_{0}$ of Borel sets such that

(i) each $B \in \mathcal{B}_{0}$ is invariant;

(ii) for each $B \in \mathcal{B}_{0}$ there exists $\alpha<\lambda$ such that $B \in \sum_{\alpha}^{0}$;

(iii) $G \cdot x=\left\{y \in X: \forall B \in \mathcal{B}_{0}(y \in B \Leftrightarrow x \in B)\right\}$. 
Proof. Let $\tau$ be the topology on $X$. Suppose $G \cdot x=\bigcap_{n \in \mathbb{N}} \bigcup_{m \in \mathbb{N}} B_{m, n}$, where each $B_{m, n}$ is $\sum_{\sim \alpha}^{0}$ for some $\alpha<\lambda$. Then following [BeKe2], we can find a countable collection $\mathcal{B}$ of Borel sets in $X$ such that

(i) for all $B \in \mathcal{B}, U \subseteq G$ basic open, $B^{* U}$ and $B^{\Delta U}$ are in $\mathcal{B}$;

(ii) for all $n, m \in \mathbb{N}, B_{m, n} \in \mathcal{B}$;

(iii) for all $B \in \mathcal{B}$ there exists $\alpha<\lambda$ such that $B \in \sum_{\alpha}^{0}$;

(iv) $\left\{B^{\Delta U}: U \subseteq G\right.$ open $\}=\mathcal{B}_{1}$ generates a Polish topology, $\tau_{0}$, on $X$ that refines $\tau$.

Fix a countable basis $\mathcal{A}$ for the topology on $G$. It follows from [Va] that

$$
(G \cdot x)^{* G}=G \cdot x=\bigcap_{\substack{n \in \mathbb{N} \\ U \in \mathcal{A} \\ U \neq \emptyset}} \bigcup\left\{B_{m, n}^{\Delta U}: m \in \mathbb{N}\right\} .
$$

So we have that $G \cdot x$ is $G_{\delta}$ in $\left(X, \tau_{0}\right)$. Hence, by 3.5 , for all $y$ in $X, G \cdot x=G \cdot y$ if and only if they have the same closure with respect to $\tau_{0}$. Thus, since $\mathcal{B}_{1}$ is a basis for $\tau_{0}$, we have that for all $y \in X$

$$
G \cdot x=G \cdot y \Leftrightarrow \forall B \in \mathcal{B}_{1}(G \cdot x \cap B \neq \emptyset \Leftrightarrow G \cdot y \cap B \neq \emptyset) ;
$$

Since the action of $G$ is continuous with respect to $\tau_{0}$, this is equivalent to

$$
\forall B \in \mathcal{B}_{1}\left(x \in B^{\Delta G} \Leftrightarrow y \in B^{\Delta G}\right) .
$$

Thus $\mathcal{B}_{0}=\left\{B^{\Delta G}: B \in \mathcal{B}_{1}\right\}$ is as required. $\prod_{\lambda+1}^{0}$.

The next corollary states that this already implies that no orbit can be properly

3.9 Corollary. Let $G$ be a Polish group that is nilpotent or has an invariant metric. Let $X$ be a Polish $G$-space. Let $x \in X$. Let $\lambda$ be a countably infinite limit ordinal. Then $G \cdot x \in \underset{\sim}{\prod_{\lambda+1}^{0}}$ implies $G \cdot x \in \underset{\sim}{\prod_{\lambda}^{0}}$.

Proof. Suppose instead that $G \cdot x \in \prod_{\lambda+1}^{0} \backslash \underset{\sim}{\prod_{\lambda}^{0}}$. Fix $\mathcal{B}_{0}$ as in 3.8. Note that the collection of $y \in X$ such that $\forall B \in \mathcal{B}_{0}(y \in B \Leftrightarrow x \in B)$ is $\prod_{\lambda}^{0}$. Hence there is a $y$ in this collection with $G \cdot x \neq G \cdot y$. However, this contradicts 3.8.

More recently it has been shown in $[\mathrm{HjKeLo}]$ that for any $\lambda$ as above we can have an abelian Polish $G$ and a Polish $G$-space $X$ such that for some $x \in X$ it is the case that $G \cdot x$ is properly $\sum_{\sim+1}^{0}$ in the sense that $G \cdot x \in \sum_{\lambda+1}^{0} \backslash \underset{\sim}{\prod_{+1}}{ }_{\lambda+1}$. We will note in section 4 that one can have an orbit that is properly $G_{\delta}$, for an abelian Polish group action.

\section{SOME EXAMPLES AND COUNTEREXAMPLES}

4.1 Example. Here we present an example of a Polish group and a continuous action of this group on a Polish space which does not fulfill the Glimm-Effros dichotomy. Actually, the dichotomy is violated in the strongest possible form: the action has two orbits both of which are dense. The group and the action are simple enough to refute some natural generalizations of Theorems 3.1 and 3.6. As was shown by these results, the Glimm-Effros dichotomy holds for

(i) actions of nilpotent Polish groups; 
(ii) actions of Polish groups admitting an invariant metric;

(iii) actions with compact stabilizers of Polish solvable groups;

(iv) actions with compact stabilizers of Polish groups admitting left invariant complete metrics.

(v) actions of locally compact Polish groups (this was already proved in [Ef]).

The following example shows that we cannot replace nilpotent by solvable in (i), admitting an invariant metric or locally compact by admitting a left invariant complete metric in (ii) and (v) respectively, and compact stabilizers by locally compact stabilizers in (iii) and (iv).

Let $G=\mathbb{Z} \times \mathbb{Z}^{\mathbb{Z}}$ with the product topology, where $\mathbb{Z}$ is given the discrete topology and $\mathbb{Z}^{\mathbb{Z}}$ the product topology. Define the multiplication in $G$ by

$$
\left(m,\left(a_{k}\right)_{k \in \mathbb{Z}}\right) \cdot\left(n,\left(b_{k}\right)_{k \in \mathbb{Z}}\right)=\left(m+n,\left(c_{k}\right)_{k \in \mathbb{Z}}\right) \text {, where } c_{k}=a_{k}+b_{k-m} .
$$

It is easy to see that $G$ with this multiplication is a topological group. Since the topology is Polish, $G$ is a Polish group. (Actually, $G$ is $\mathbb{Z} \times_{\varphi} \mathbb{Z}^{\mathbb{Z}}$ for a suitable homomorphism $\varphi: \mathbb{Z} \rightarrow \operatorname{Aut}\left(\mathbb{Z}^{\mathbb{Z}}\right)$.) Note that $H=\left\{\left(0,\left(a_{k}\right)_{k \in \mathbb{Z}}\right): a_{k} \in \mathbb{Z}\right\}$ is a clopen abelian subgroup of $G$. Note also that $H \triangleleft G$. Thus, since $G / H \cong \mathbb{Z}$,

(1) $G$ is solvable of rank 2 .

Claim. Let $G$ be a Polish group containing a clopen abelian subgroup $H$. Then $G$ admits a left invariant complete metric.

Proof of Claim. Let $d$ be a left invariant metric on $G$, which exists by the Birkhoff-Kakutani theorem (see 9.1 of [Ke1]). We need to show that $d$ is complete. Note that since $H$ is abelian and Polish, $d \uparrow H$ is complete. Also, since $H$ is open, there is $\delta>0$ such that if $d(g, 1)<\delta$, then $g \in H$. Now, let $\left(g_{n}\right)_{n \in \mathbb{N}}$ be a $d$-Cauchy sequence. Find $N \in \mathbb{N}$ with $d\left(g_{n}, g_{m}\right)<\delta$ for $n, m \geq N$. Since $d$ is left invariant, $d\left(g_{N}^{-1} g_{n}, 1\right)<\delta$, so $g_{N}^{-1} g_{n} \in H$ for $n \geq N$. But again by the left invariance of $d$, the sequence $\left(g_{N}^{-1} g_{n}\right)_{n \in \mathbb{N}}$ is $d$-Cauchy. Thus there is $g \in H$ with $g_{N}^{-1} g_{n} \underset{n}{\longrightarrow} g$. Hence $g_{n} \underset{n}{\longrightarrow} g_{N} g$. It follows that $d$ is complete.

As an immediate consequence of the claim we get

(2) $G$ admits a left invariant complete metric.

Now we define an action of $G$. Let $A$ be the one point compactification of $\mathbb{Z}$, i.e., $A=\mathbb{Z} \cup\{\infty\}$. For $n \in \mathbb{Z}$, define $n+\infty=\infty+n=\infty$. Define an action of $G$ on $A^{\mathbb{Z}}$ by

$$
\left(m,\left(a_{k}\right)_{k \in \mathbb{Z}}\right) \cdot\left(b_{k}\right)_{k \in \mathbb{Z}}=\left(c_{k}\right)_{k \in \mathbb{Z}}, \text { where } c_{k}=a_{k}+b_{k-m} .
$$

A straightforward calculation shows that this is indeed an action. The continuity of this action is also easy to check. Now let

$$
X=\left\{\left(a_{k}\right)_{k \in \mathbb{Z}} \in A^{\mathbb{Z}}: \forall k, \ell \in \mathbb{Z} k \neq \ell \Rightarrow\left(a_{k} \neq \infty \text { or } a_{\ell} \neq \infty\right)\right\} .
$$

Clearly $X$ is a $G_{\delta}$ in $A^{\mathbb{Z}}$ and is $G$-invariant. Thus, $X$ is a Polish $G$-space. Note that $X$ is the union of two orbits, $G \cdot(\cdots, 0,0,0, \cdots)$ and $G \cdot(\cdots, 0,0, \infty, 0,0, \cdots)$, both of which are dense. Thus,

(3) Neither $E_{0} \sqsubseteq_{c} E_{G}^{X}$ nor $E_{G}^{X} \in G_{\delta}$.

The stabilizers of points in $X$ are isomorphic to $\mathbb{Z}$, hence

(4) $G_{x}$ is locally compact for all $x \in X$.

4.2 Example. The proof of 3.9 reduced the situation of a $\prod_{\lambda+1}^{0}$ orbit that is not $\prod_{\lambda}^{0}$ to the situation of a $G_{\delta}$ orbit that has another orbit with the same closure, and 
this in turn was noted to be inconsistent with 3.1. Therefore we observe that a variation of 4.1 shows that it is possible to have an abelian Polish $G$ and a properly $G_{\delta}$ orbit in some Polish $G$-space.

Let $\mathbb{Z}^{\mathbb{Z}}$ act on $A^{\mathbb{Z}}$ as in 4.1 , so that $(g \cdot x)(n)=x(n)+g(n)$ for any $n \in \mathbb{Z}, g \in$ $Z^{\mathbb{Z}}, x \in A^{\mathbb{Z}}$. Fix any $x \in A^{\mathbb{Z}}$ such that $\forall n \in \mathbb{Z}(x(n) \neq \infty)$. It follows from the definition of the action that $G \cdot x=\left\{y \in A^{\mathbb{Z}}: \forall n \in \mathbb{Z}(y(n) \neq \infty)\right\}$ and is a dense $G_{\delta}$. We just need to check that $G \cdot x \notin F_{\sigma}$.

But if $G \cdot x=\bigcup_{n \in \mathbb{N}} B_{n}$, each $B_{n}$ closed, then by the Baire category theorem some $B_{n}$ must contain an open set. So $G \cdot x$ contains an open set, and hence the orbit is open. However, this conclusion is easily seen to be untrue.

The next example is a simplified variation of a construction given at the end of [Ben] for the similar purpose of showing that Vaught's two cardinal theorem fails for $\omega$-logic.

4.3 Example. Let $\mathcal{L}$ be the first order language generated by the binary symbol $<$ and the constants $\left\{c_{q}: q \in \mathbb{Q}, q \neq 0\right\}$. Let $X_{\ell}$ be the Polish space of countable $\mathcal{L}$-structures with universe $\mathbb{N}$, equipped with the topology generated by first order logic. So the basic open sets consist exactly of sets of the form

$$
\left\{M \in X_{\ell}: M \models \varphi\left(n_{0}, \cdots, n_{\ell}\right)\right\}
$$

for some $\varphi \in \mathcal{L}$ and $n_{0}, \cdots, n_{\ell} \in \mathbb{N}$. We let $S_{\infty}$ act on $X_{\mathcal{L}}$ by the specification that

$$
g \cdot M \models " n_{0}<n_{1} " \Leftrightarrow M \models " g^{-1}\left(n_{0}\right)<g^{-1}\left(n_{i}\right) "
$$

and that for $q \in \mathbb{Q}, q \neq 0$,

$$
\left(c_{q}\right)^{g \cdot M}=g\left(\left(c_{q}\right)^{M}\right) .
$$

The fact that $X_{\mathcal{L}}$ becomes a Polish $S_{\infty}$-space under this topology and this action is easily checked, and in fact was proved in [GrMoRy].

Let $\mathcal{M}$ be the model with universe $\mathbb{Q}$, where we have for each $q \in \mathbb{Q}, q \neq 0$,

$$
\left(c_{q}\right)^{\mathcal{M}}=q
$$

and for each $q_{1}, q_{2} \in \mathbb{Q}$

$$
\mathcal{M}=" q_{1}<q_{2} " \Leftrightarrow q_{1}<q_{2} .
$$

Let $\mathcal{N}$ be the substructure of $\mathcal{M}$ consisting of $\{q \in \mathbb{Q}: q \neq 0\}$. Let $T$ be equal to $\operatorname{Th}_{\mathcal{L}} \mathcal{M}=\{\varphi \in \mathcal{L}: \mathcal{M} \models \varphi\}$. It follows easily from the quantifier elimination theorem for dense linear orderings, as found in [ChKe], that $\mathcal{N} \models T$; the key point here is that any formula $\varphi \in \mathcal{L}$ contains only finitely many of the constants $\left\{c_{q}: q \in \mathbb{Q}, q \neq 0\right\}$. Note that $\left\{M \in X_{\mathcal{L}}: M \models T\right\}$ is a closed set in $X_{\mathcal{L}}$, and hence Polish.

Let $C$ consist of the collection of $M \in X_{\mathcal{L}}$ such that $M \models T$ and

$$
\forall n \in \mathbb{N} \forall k \in \mathbb{N} \exists q \in \mathbb{Q}, q \neq 0\left(M \models \text { " } c_{1 / k}>n>c_{-1 / k} \vee n=c_{q} "\right) .
$$

It is easily seen that the following all hold:

(a) $C \in G_{\delta}$, and is $S_{\infty}$-invariant; hence,

(b) $C$ is a Polish $S_{\infty}$-space;

(c) $C$ has exactly two orbits, corresponding to the isomorphism types of $\mathcal{M}$ and $\mathcal{N}$ above; therefore, since $\left\{c_{q}: q \in \mathbb{Q}, q \neq 0\right\}$ are dense in both $\mathcal{M}$ and $\mathcal{N}$,

(d) the action of $S_{\infty}$ on $C$ is free; 
(e) both orbits are dense.

Thus we have a Polish $S_{\infty}$-space where the action is free, the action is minimal, $E_{S_{\infty}}^{C}$ is not $G_{\delta}$, and yet we do not have $E_{0} \sqsubseteq E_{S_{\infty}}^{C}$.

One can also obtain a more topological example of a minimal free action with exactly two orbits.

4.4. Example. Let $\mathcal{N}$ be the collection of functions from $\mathbb{N}$ to $\mathbb{N}$ equipped with the product topology we obtain by viewing $\mathbb{N}$ as a discrete space. $\mathcal{N}$ is Polish.

Let $X_{0} \subseteq \mathcal{N}$ consist of those functions $f: \mathbb{N} \rightarrow \mathbb{N}$ that are one to one. $X_{0}$ is closed in $\mathcal{N}$, so it is again Polish. Let $S_{\infty}$ act on $X_{0}$ by left composition. Note that this is a continuous action, and for any $f_{0}, f_{1} \in X_{0}$

$$
f_{0} E_{X_{0}}^{S_{\infty}} f_{1} \Leftrightarrow\left|\mathbb{N}-\operatorname{Ran}\left(f_{0}\right)\right|=\left|\mathbb{N}-\operatorname{Ran}\left(f_{1}\right)\right| .
$$

Actually it is not hard to show that if $d_{\ell}$ is any compatible left invariant metric on $S_{\infty}$, and $\left(\hat{S}_{\infty}, d_{\ell}\right)$ is its Cauchy completion, then $\hat{S}_{\infty}$ and $X_{0}$ are isomorphic as Polish $S_{\infty}$-spaces. However, we have no need of this fact.

Now let $X \subseteq X_{0}$ consist of all $f \in X_{0}$ such that

$$
|\mathbb{N}-\operatorname{Ran}(f)| \leq 1 \text {. }
$$

It is easily checked that $X$ is $G_{\delta}$ in $X_{0}$, and hence it is again a Polish $S_{\infty}$-space. By our above criteria, $X$ has exactly two orbits. It is routine to show that the action is minimal and free.

We finished the first draft of the paper with three open problems for solvable Polish groups. Note that this class of Polish groups is included in the class of Polish groups allowing a complete left invariant metric, either by the techniques of section 3 or by the much more direct argument of [Ga]. Following work in early 1996 by Howard Becker, these were all answered affirmatively. First, Becker showed in $[\mathrm{Be}]$ that $\operatorname{TVC}(G)$ holds for all Polish groups with a complete left invariant metric. He also showed $\operatorname{TVC}\left(G, \Sigma_{1}^{1}\right)$ under large cardinal or determinacy assumptions, and

then later $[\mathrm{Hj}]$ proved this in just ZFC.

Finally, while we have already seen that the Glimm-Effros property as formulated above fails for solvable groups, Becker showed that if $G$ is a complete left invariant Polish group and $X$ a Polish $G$-space then we must have either

I) $E_{0} \sqsubseteq c E_{G}^{X}$, or

II) $\exists f: X \rightarrow 2^{\omega}, f \in \Delta_{\sim}^{1} \forall x, y \in X\left(x E_{G}^{X} y \Leftrightarrow f(x)=f(y)\right)$.

\section{REFERENCES}

[Be] H. Becker, Vaught's conjecture for complete left invariant Polish groups, handwritten notes, University of South Carolina, 1996.

[Ben] M. Benda, Remarks on countable models, Fundamenta Mathematicae, vol. 81 (1974), pp. 107-118. MR 51:7852

[BeKe1] H. Becker and A. S. Kechris, Borel actions of Polish groups, Bulletin of the American Mathematical Society, vol. 28 (1993), pp. 334-341. MR 93m:03083

[BeKe2] H. Becker and A. S. Kechris, The descriptive set theory of Polish groups actions, Cambridge, London Mathematical Society Lecture Note Series, 1997. MR 98d:54068

[ChKe] C. C. Chang and H. J. Keisler, Model theory, Amsterdam, North-Holland, 1973. MR 53: 12927

[Ef] E. G. Effros, Polish transformation groups and classification problems, General topology and modern analysis, Rao and McAuley (eds.), New York, Academic Press, 1981, pp. 217-227. MR 82k:54064 
[Ga] S. Gao, Automorphism groups of countable structures, Journal of Symbolic Logic, vol. 63 (1998), pp. 891-896.

[Gl] J. Glimm, Locally compact transformation groups, Transactions of the American Mathematical Society, vol. 101 (1961), pp. 124-138. MR 25:146

[GrMoRy] A. Grzegorczyk, A. Mostowski, C. Ryll-Nardzewski, Definability of sets of models of axiomatic theories, Bulletin of the Polish Academy of Sciences (Mathematics, Astronomy and Physics), vol. 9 (1961), pp. 163-167. MR 29:1138

[Ha] L. Harrington, Analytic determinacy and 0\#, Journal of Symbolic Logic, vol. 43 (1978), pp. 685-694. MR 80b:03065

[HaKeLo] L. Harrington, A. S. Kechris, A. Louveau, A Glimm-Effros dichotomy theorem for Borel equivalence relations, Journal of the American Mathematical Society, vol. 3 (1990), pp. 903-928. MR 91h:28023

[HaSa] L. Harrington and R. Sami, Equivalence relations, projective and beyond, Logic Colloquium'78, Amsterdam, North-Holland, 1979, pp. 247-264. MR 82d:03080

[Hj] G. Hjorth, Orbit cardinals, preprint, UCLA, 1996.

[HjKe] G. Hjorth and A. S. Kechris, Analytic equivalence relations and Ulm-type classifications, Journal of Symbolic Logic., vol. 60(1995), pp. 1273-1300. MR 96m:54068

[HjKeLo] G. Hjorth, A.S. Kechris, and A. Louvaeu, Borel equivalence relations induced by actions of the symmetric group, Annals of Pure and Applied Logic, vol. 92 (1998), pp. 63-112. CMP 98:13

[Ke1] A. S. Kechris, Classical descriptive set theory, New York, Springer-Verlag, 1995. MR 96e:05057

[Ke2] A. S. Kechris, Lectures on definable group actions and equivalence relations, unpublished manuscript, Los Angeles, 1994.

[Mi] A. Miller, On the Borel classification of the isomorphism type of a countable model, Notre Dame Journal of Formal Logic, vol. 24 (1983), pp. 22-34. MR 84c:03055

[MiD] D. Miller, On the measurability of orbits in Borel actions, Proceedings of the American Mathematical Society, vol. 63(1977), pp. 165-70. MR 55:13394

[Mo] Y. N. Moschovakis, Descriptive set theory, Amsterdam, North-Holland, 1980. MR 82e:03002

[Sa] R. L. Sami, Polish group actions and the Vaught conjecture, Transactions of the American Mathematical Society, vol. 341 (1994), pp. 335-353. MR 94c:03068

[Si] J. H. Silver, Counting the number of equivalence classes of Borel and co-analytic equivalence relations, Annals of Mathematical Logic, vol. 18 (1980), pp. 1-28. MR 81d:03051

[So] S. Solecki, Equivalence relations induced by actions of Polish groups, Transactions of the American Mathematical Society, 347 (1995), pp. 4765-4777. MR 96c:03100

[Va] R. L. Vaught, Invariant sets in topology and logic, Fundamenta Mathematicae, vol. 82 (1974), pp. 269-293. MR 51:167

Department of Mathematics, 253-37, California Institute of Technology, Pasadena, California 91125

Current address: Department of Mathematics, MSB 6363, University of California, Los Angeles, California 90095-1555

E-mail address: greg@math.ucla.edu

Department of Mathematics, Indiana University, Bloomington, Indiana 47405

E-mail address: ssolecki@indiana.edu 